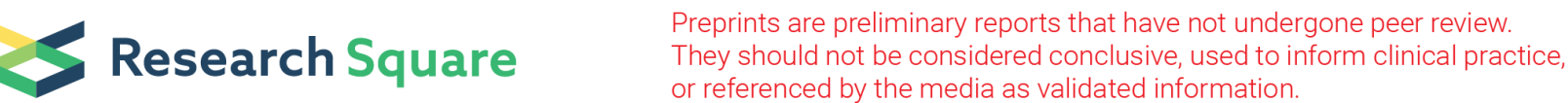

\section{Protecting Clinical Trials in Cystic Fibrosis; COVID- 19 Mitigation Measures Crowd Sourced from the European Cystic Fibrosis Society Clinical Trials Network}

Silke van Koningsbruggen-Rietschel ( $\sim$ Silke.vanKoningsbruggen@uk-koeln.de )

University Hospital Cologne: Uniklinik Koln https://orcid.org/0000-0002-2222-4238

Fiona Dunlevy

European Cystic Fibrosis Society

Veerle Bulteel

European Cystic Fibrosis Society

Kate Hayes

Queen's University Belfast

Anne Verbrugge

European Cystic Fibrosis Society

Hettie M. Janssens

Erasmus MC Sophia Children Hospital: Erasmus MC Sophia

Nadine Dufeu

Aix-Marseille-University: Aix-Marseille Universite

Nicolas J. Simmonds

Royal Brompton Hospital

Lieven J. Dupont

KU Leuven University Hospitals Leuven: Katholieke Universiteit Leuven Universitaire Ziekenhuizen

Leuven

Damien G. Downey

Queen's University Belfast

\section{Research Article}

Keywords: pandemic, mitigation, SARS-CoV-2, Covid-19, clinical trials, cystic fibrosis

Posted Date: April 28th, 2021

DOI: https://doi.org/10.21203/rs.3.rs-297415/v1 
License: (c) (i) This work is licensed under a Creative Commons Attribution 4.0 International License. Read Full License 


\section{Abstract}

The SARS-CoV-2 pandemic has disrupted clinical trials worldwide. The European Cystic Fibrosis SocietyClinical Trials Network (ECFS-CTN) has tracked clinical trial disruption by surveying its 58 trial sites across 17 European countries and collated information on measures to mitigate the impact of the pandemic and ensure trial continuity.

Here we present recommendations on how to reduce the risk of SARS-CoV-2 exposure to patients and trial staff by implementing remote trial visits where possible, using home assessments, video and phone calls, electronic consent and home delivery of study drug. We discuss the practicalities of remote source data verification, protocol amendments, changing trial site location, and staff absences and home working. We outline how to protect trial outcomes, including home assessments, safety reporting, protocol deviations and recruitment challenges. Finally, we discuss the importance of continued access to study drug via extension trials for some patients.

This guidance was co-created from the shared knowledge and experience of sites in our network and was re-distributed directly to all ECFS-CTN sites to help mitigate the impact of further waves of the SARS-CoV2 pandemic. We will also use this guidance to assist companies, academia and consortia with future protocol design and risk mitigation plans.

\section{Background}

The Severe Acute Respiratory Syndrome Coronavirus 2 (SARS-CoV-2) pandemic has disrupted clinical trial conduct and setup worldwide, largely due to mitigation efforts including self-isolation and reorganization of hospitals and staff to handle escalating admissions of infectious patients. Clinical trial teams, hospitals, sponsors as well as competent authorities have scrambled to adapt to the challenges posed by the pandemic. The overriding concern is protection of clinical trial participants and healthcare professionals involved in trial conduct. The FDA issued regulatory guidance for sponsors in mid-March 2020 (1), closely followed by EMA guidance a few days later (2). Guidance for ongoing trials includes switching to telephone/remote visits when possible, extending trial duration, home delivery of study drug, and remote monitoring. These documents also provide guidance on protocol amendments, updating informed consent, documenting protocol deviations, and handling missing data after trial completion. Sponsors are advised to assess the feasibility of starting new trials and enrolling new patients into ongoing trials.

Since 2009, the European Cystic Fibrosis Society-Clinical Trials Network (ECFS-CTN) has been working to improve the quality and conduct of clinical trials in the rare disease cystic fibrosis (CF), with the aim of accelerating the availability of new therapies. We currently federate 58 trial sites across 17 countries in Europe. Our work includes review of study protocols and investigator brochures, development and standardization of clinical trial measures and outcomes, training of investigators and research coordinators and quality assurance of trial sites (3). 
As SARS-CoV-2 spread across Europe in early 2020, it became clear that the crisis would impact the conduct of clinical trials in CF. We started regularly surveying our member sites to understand the impact on CF trials across Europe. The results of each survey were promptly returned to sites and partner patient organizations. We described the methodology and survey results previously (4).

Over the summer we took advantage of the lull in SARS-CoV-2 infections to review the survey results and extract information about the mitigation measures that sites had implemented to ensure continuity of clinical research. We then combined the information into a structured document and circulated it to all network sites for comment.

Our collaborative approach allowed us to source the best mitigation practices from all over Europe, thereby providing our member sites with useful guidance that could be locally adapted to mitigate the effects of the pandemic on clinical research and ensure continuity of existing and new trials for CF treatments.

We identified 15 areas of risk that could be impacted by resurgences of Covid-19 (Table 1). Here, we present our recommended mitigations measures for each risk. These recommendations are not binding; rather they provide sites with a list of items to consider and to potentially adapt to their local situation and institutional guidelines, along with any protocol amendments for specific studies. 
Table 1

Risks to clinical trials in cystic fibrosis

\section{Preventing \\ SARS-CoV-2 \\ infection}

Covid-19

Patients could be infected or have been exposed to SARS-CoV-2.

infection

Physical site visit A physical visit at the site is not possible/inadvisable due to the risk of infection.

Consenting of Informed consent process may not be possible if patients are not allowed to

patients

attend trial visits in clinic.

Study drug

Study drug cannot be provided if the patient is not allowed to come to the site.

Logistics

Source data

verification

SDV needs to be performed remotely if monitors are not allowed to come to the

(SDV)

Handling

protocol trial site.

amendments

Changing location of trial site

Mitigation measures may lead to more protocol amendments than usual.

Staff absence
Staff working
from home

Clinical staff (investigators, nurses etc.) may fall ill, have to be quarantined, be reassigned to clinical duties or Covid-19 clinical trials.

Reorganization of hospitals may mean that the trial site is effectively moved to another location within the hospital or even to another hospital.

\section{Protecting trial}

outcomes

Assessments

Assessments cannot be done at the site due to the risk of infection.

Compliance

check

Home working policies and effective information technology solutions are needed to cater for staff forced to work from home.

Increase in adverse events

Patients are not coming to the site, compliance on returned study drug packaging cannot be verified.

Protocol deviations

Occurrence of adverse events may increase during the pandemic.

Low recruitment

Due to pandemic restrictions, there may be more protocol deviations.

Recruitment may be below expectations, expected patients drop out or/and new patients are not allowed to come to the clinic.

Access to study

drug 
Preventing

SARS-CoV-2

infection

Initiating rollover Blanket bans on new trial initiation may block rollover trials and continued trials access to study drug.

\section{Preventing Sars-cov-2 Infection}

Ensuring the safety of study participants and staff is key. Patients may be wary of coming to clinic for trial visits and becoming infected. Conversely, they could be asymptomatic or pre-symptomatic transmitters of SARS-CoV-2 and infect staff when they come to clinic.

\section{Pre-screening for SARS-CoV-2 before trial visits}

We recommend that before patients attend trial visits in clinic, they are pre-screened for Covid-19 associated symptoms via email, telephone or a video call. Alternatively, they could provide a recently negative SARS-CoV-2 PCR test result ( $\leq 24$ to 48 hours before the planned visit), especially if the trial visit involves aerosol-generating procedures. Self-administered rapid antigen diagnostic tests may be permitted in some countries and institutions, but PCR-testing remains the gold standard.

\section{Electronic consent}

The use of electronic consent (eConsent) can facilitate remote clinical assessments. Several apps and platforms exist for electronic consent, however regulations governing their use vary by country and even by institution. Use of eConsent platforms may need to be approved by local ethics committees. Sites should check if study sponsors can offer technical solutions for eConsent.

\section{Shipping study drug}

The shipment of study drug directly to patients' homes can also reduce clinic contact time. Again, not all national and local regulations allow this. Patients may need to be educated on proper storage of study drug and administration. Temperature requirements for transport of study drug must also be considered.

\section{Telephone/video visits}

If local travel restrictions are in place, onsite trial visits can be minimized if they can be performed by telephone or video call using safe platforms. Some countries allow home visits by qualified personnel. Clinical trial visits could be scheduled and combined with routine clinical care visits to reduce the risk of infection if patients need to be seen at the trial site. Face-to-face site visits should only occur if they cannot be performed remotely. Sites should prioritize visits that assess primary outcomes and important safety parameters, e.g. liver function tests. Patients attending onsite visits should be offered the possibility to come by private taxi if their only alternative is public transport. Sites and clinical trial networks should advocate that sponsors cover these extended travel costs. 


\section{Logistics}

\section{Remote source data verification}

Many hospitals have suspended the possibility of external visitors such as site monitors (4), forcing sponsors to switch to remote source data verification (SDV). Sites in countries that allow SDV should work with their sponsor and contract research organization (CRO) to determine what information can be shared and how, while remaining compliant with data protection regulations. We previously observed that remote SDV is more time-consuming than face-to-face SDV (4). This could be dealt with by a budget amendment, with costs covered directly by the commercial sponsor. Proactive planning can help sites handle the increased workload of "catch-up" monitoring that will occur when restrictions are lifted. Site staff may require training on remote SDV procedures. A protocol amendment (and ethics committee approval) may be required.

\section{Protocol amendments}

Protocol amendments are likely for ongoing trials. If sites do not already have standard workflows for handling protocol amendments, they should plan workflows for gaining approval (from ethics committees, competent authorities and local committees), training site staff and re-consenting participants, if necessary.

\section{Moving the trial site}

In some areas, hospital services have been completely reorganized to separate Covid-19 and non-Covid19 services. Changing the physical location of a trial site to another part of the same hospital, or even to a different hospital can help reduce the risk of exposing trial participants to SARS-CoV-2, but requires significant preparation, both physical and administrative. Clear communication with the entire clinical trial team is critical and the date of the relocation should be communicated to the patient, sponsor, and other relevant partners. Any equipment moved will need to be recalibrated. Fridges and freezers will need to be equipped with alarms systems for temperature monitoring. Access to time-sensitive consumables such as dry ice should be verified before any patient visits. Couriers should be notified of any change in address for sample pickup. If applicable, pharmacy staff should be involved while planning the move to check whether study drug can be distributed to the new location (if home delivery of study drug has not already been established). The new trial location should be equipped with locked cabinets to protect patient and trial data and any relocation of files, study material, storage conditions, changes in equipment, etc. should be documented. Any new personnel associated with the move should be trained appropriately and added to the delegation log. Finally, the local ethics committee should be notified of the change in location.

\section{Staff quarantine \& home working}

Staffing levels can be reduced if clinical trial staff (research coordinators, nurses and investigators) are reassigned to Covid-19 clinical duties or trials, fall ill, or have close contact with a confirmed case, leading 
to obligatory isolation at home (4).

If clinical research duties must be delegated to other team members, they should undergo the relevant training (good clinical practice [GCP], protocol-specific etc.). All personnel changes should be documented in delegation and training logs. In some cases, the local ethics committee may need to be notified, particularly if the principal investigator changes.

Some trial staff, who have more administrative, regulatory or data management roles, may be forced by institutional regulations to work from home to reduce exposure to the virus. Sites and their institutions should develop home working policies as a priority, with technical solutions such as providing homebased staff with encrypted computers and secure networks to access documents. Home working policies should provide staff with guidance on data protection.

The pandemic may also disrupt the clinical trial supply chain, creating a shortage of ancillary supplies for clinical trials. Sites should be aware of their inventory of clinical trials related materials and should interact with sponsors and CRO to adequately forecast patient recruitment.

\section{Finances}

The SARS-CoV-2 pandemic and the mitigations measures suggested in this paper will invariably have a financial impact. We have focused on industry-sponsored trials here, and advise sites to discuss the cost of mitigation measures upfront with sponsors. However, many trials are investigator-initiated and funded by academia or grants from charities, national research bodies or European programs. We advise sites participating in such trials to immediately open communications with the trial funder to discuss extra funding for mitigating the impact of the pandemic, or for grant extensions to account for delays caused by the pandemic.

\section{Protecting Trial Outcomes}

Pandemic-related disruption of ongoing trials could prevent the robust assessment of outcomes, in particular safety and the primary outcome. It is critical to minimize trial disruption to preserve the integrity of ongoing trials, and to avoid the delayed introduction of potentially life-extending medicines. This is particularly important for life-threatening rare diseases with limited treatment options such as CF. It is also an ethical obligation to ensure that trials avoid research waste by adequately answering the research question.

\section{Assessments}

Assessments underpinning the primary endpoint should be prioritized. If sponsors do not proactively provide guidance, sites can ask sponsors to provide a minimal core data set (plan "B") outlining which outcome measures can be obtained remotely where possible. 
If patients are provided with the relevant sterile equipment and instructions (written, by telephone or video), they can provide biological samples (e.g. urine, sputum) from home, which can be collected by courier and transported to the hospital. Other common assessments that can be performed at home include filling in any patient-reported outcome questionnaires, pregnancy testing, height and weight (ideally using equipment provided and calibrated by the sponsor). This will require close collaboration with the sponsor and consideration of extra costs. Ethics approval could be needed for such amendments.

Some assessments can be performed by private home-care services, hired by the sponsor, or in some cases, the site research nurse or coordinator. These include electrocardiogram, blood draws, vital signs, and pulse oximetry. Case report forms may need to be modified to account for such changes.

Spirometry is a key assessment parameter in CF and is often a primary outcome in clinical trials. Patients require training to perform this maneuver, therefore, the results are highly dependant on active cooperation of the patients. With recent technological advances, compact home spirometry equipment is now available, but is not yet implemented as standard in routine CF care. In addition, these devices have not yet been validated for assessment of spirometry endpoints for clinical trials. In face-to-face spirometry assessments, patients are coached by the research nurse or coordinator regarding the necessary technique required to perform a "valid" forced expiratory maneuver. Spirometry performed at home, even when utilizing remote coaching via video-link may therefore be qualitatively suboptimal as compared to an in-clinic assessment. This could lead to variable results, especially if some assessments are performed at home and others in clinic. A study sponsored by the Cystic Fibrosis Foundation is investigating how home spirometry results can be "corrected" in order to be comparable to spirometry performed in clinic (5). It is unknown at this point how regulators will evaluate the results of key outcome parameters obtained with unvalidated home equipment.

\section{Study drug compliance}

Checking compliance is an important way to ensure that patients are taking study drug as instructed. This is often monitored by the patients returning empty study drug packaging to the trial site. If patients are not attending clinic, they cannot bring back empty study drug packaging to assess compliance. Alternative ways to document compliance include having the patient photograph packages of used and unused study drug packaging (blister packs) and send these photographs to the site or check online during video call and take pictures (screen shots) of patients demonstrating used and unused packages. Sites can also ask patients to collect all packaging to return to site when mitigation measures are no longer in place or can organize pick-up at home.

\section{Adverse events}

If trial participants become infected with SARS-CoV-2, there may be a rise in the number of adverse events (AEs) and serious adverse events (SAEs). Sites should discuss with the sponsor how to handle these AEs/SAEs. Patients should be reminded to call the trial team in case of any possible AEs. Phone/video calls can be performed to collect and record concomitant medications and AEs. 


\section{Protocol deviations}

Similarly, restrictions related to the SARS-CoV-2 pandemic may lead to more protocol deviations, especially around missed visits and assessments. Sites should discuss how to handle, and report protocol deviations related to the pandemic prior to database lock with sponsors.

\section{Recruitment}

The pandemic may impact recruitment since patients may be more hesitant to enroll in new trials, may drop out of ongoing trials, or hospital rules may prevent patients from joining new trials. Sites should discuss with the sponsor in advance under which circumstances the study will be stopped due to low recruitment or whether the recruitment window can be extended.

\section{Continued Access To Study Drug}

Blanket bans on new trial initiation may block extension/rollover trials and continued access to investigational drugs. This is particularly important for patients for whom highly effective therapies do not exist or are not accessible. For the rare disease $\mathrm{CF}$, we were concerned that this would block continued access to a new class of medicines called cystic fibrosis transmembrane conductance regulator (CFTR) modulators. We previously reported that most ECFS-CTN sites were allowing extension trials to start as planned, ensuring access to these study drugs (4).

Investigators, learned societies, clinical trial networks and patient organizations can advocate for these trials to be treated as high-priority trials.

\section{Conclusions}

These recommendations were co-created from the shared knowledge and experience of sites in our network and were subsequently distributed directly to all ECFS-CTN sites. We will also use this guidance to assist companies, academia and consortia with future protocol design and risk mitigation plans. Indeed, many of the mitigation measures proposed in this manuscript are difficult to implement at site level without the logistical and financial support of the study sponsor. We also feel that industry, regulators, $\mathrm{CF}$ clinical trial networks and investigative sites, with the help of the patient organizations and with the experience gained during the SARS-CoV-2 pandemic could consider adopting virtual and remote technologies to promote improved patient-centricity in future clinical trials. Indeed, the rapid pivot towards home monitoring and other remote measures may pave the way for a shift towards more "remote" trial visits in the future. We hope that our manuscript might help trial sites who are not otherwise supported by a clinical trials network, and who would benefit from the collective knowledge and experience of our ECFS-CTN member sites. We also hope that our manuscript will serve as a starting point for continued conversation as well as sharing of experience and best practice.

\section{List Of Abbreviations}




$\begin{array}{ll}\text { AE } & \text { adverse event } \\ \text { CRO } & \text { contract research organization } \\ \text { CF } & \text { cystic fibrosis } \\ \text { CFTR } & \text { cystic fibrosis transmembrane conductance regulator } \\ \text { eConsent } & \text { electronic consent } \\ \text { ECFS-CTN } & \text { European Cystic Fibrosis Society-Clinical Trials Network } \\ \text { GCP } & \text { good clinical practice } \\ \text { SAE } & \text { serious adverse event } \\ \text { SARS-CoV-2 } & \text { severe acute respiratory syndrome coronavirus 2 } \\ \text { SDV } & \text { source data verification }\end{array}$

\section{Declarations}

Ethics approval and consent to participate: Not applicable.

Consent for publication: Not applicable.

Availability of data and material: Not applicable.

\section{Competing interests:}

$\mathrm{FD}, \mathrm{VB}, \mathrm{KH}$ and $\mathrm{AV}$ have no competing interests.

- SvK and LD have no relevant conflicts of interest to declare pertaining this manuscript.

- HJ has received a speaker's fee and a research grant for an investigator-initiated study from Vertex.

- NJS has received fees for consultancy and speaking from Vertex, Chiesi, Gilead, Roche, Teva, Zambon and Pulmocide.

- DD has received honoraria from Vertex and Chiesi.

Funding: Not applicable.

Authors' Contributions: SvK, FD, VB, KH and AV planned, distributed and analysed the regular surveys, and prepared the manuscript. PdC, HJ, ND, NJS, LD and DD oversaw the project and survey results and contributed to the manuscript. All authors read and approved the final manuscript. 
Acknowledgements: We acknowledge the input of clinical research teams at our 58 member sites, represented by their lead investigators: Michel Abély, Celeste Barreto, Elisabetta Bignamini, Stephanie Bui, Mary Carroll, Carlo Castellani, Raphael Chiron, Marco Cipolli, Malena Cohen, Carla Colombo, Jane Davies, Damian Downey, Lieven Dupont, Elke De Wachter, Pavel Drevinek, Jamie Duckers, Helmut Ellemunter, Isabelle Fajac, Silvia Gartner, Marita Gilljam, Adrien Halász, Charlie Haworth, Lena Hjelte, Lukas Homola, Alex Horsley, Hettie Janssens, Christiane Knoop, Tim Lee, Michael Lorenz, Vincenzia Lucidi, Gordon MacGregor, Marcus Mall, Christophe Marguet, Noel McElvaney, Ed McKone, Alexander Moeller, Susanne Nährig, Paolo Palange, Isabelle Pin, Dario Prais, Tania Pressler, Anne Prevotat, Sophie Ramel, Philippe Reix, Gernot Rohde, Gary Ruiz, Dorota Sands, Florian Singer, Alan Smyth, Olaf Sommerburg, Sivagurunathan Sutharsan, Giovanni Taccetti, Suzanne Terheggen-Lagro, Burkhard Tümmler, Eva Van Braeckel, Kors van der Ent, Silke van Koningsbruggen-Rietschel and Joanna Whitehouse.

We would like to thank Isabelle Fajac as president of ECFS for supporting this project.

\section{References}

1. Food and Drug Administration. FDA Guidance on Conduct of Clinical Trials of Medical Products during COVID-19 Pandemic. 2020 [updated May 14 2020. Available from: https://www.fda.gov/media/136238/download.

2. European Medicines Agency. Guidance on the Management of Clinical Trials during the COVID-19 (Coronavirus) pandemic. 2020 [updated April 28 2020. Available from: https://ec.europa.eu/health/sites/health/files/files/eudralex/vol10/guidanceclinicaltrials_covid19_en.pdf.

3. De Boeck K, Bulteel V, Fajac I. Disease-specific clinical trials networks: the example of cystic fibrosis. Eur J Pediatr. 2016;175(6):817-24.

4. van Koningsbruggen-Rietschel S, Dunlevy F, Bulteel V, Downey DG, Dupont L. SARS-CoV-2 disrupts clinical research: the role of a rare disease-specific trial network. Eur Respir J. 2020;56(3).

5. Paynter A, Goss C, Heltshe S, Khan U, Lechtzin N, Mayer-Hamblett N, editors. Home versus clinic spirometry to inform trial endpoints in CF: EICE experience (abstract and poster). North American Cystic Fibrosis Conference; 2020; Online. 